\title{
Publisher Correction: Interatomic force laws that evade dynamic measurement
}

John E. Sader, Barry D. Hughes, Ferdinand Huber (D) and Franz J. Giessibl (D)

Correction to: Nature Nanotechnology https://doi.org/10.1038/s41565-018-0277-x, published online 6 December 2018.

In the version of this Comment originally published, equation (4) contained the wrong inequality sign of $\lesssim$ rather than $\gtrsim$; the equation has now been corrected in the online versions of the Comment to read $S(F) \equiv \frac{z_{\text {inf }}^{2}}{4} \frac{F^{\prime \prime \prime}\left(z_{\text {inf }}\right)}{F^{\prime}\left(z_{\text {inf }}\right)} \gtrsim-1$.

Published online: 9 January 2019

https://doi.org/10.1038/s41565-019-0364-7

\section{Publisher Correction: Immunoglobulin deposition on biomolecule corona determines complement opsonization efficiency of preclinical and clinical nanoparticles}

Vivian P. Vu, Geoffrey B. Gifford, Fangfang Chen, Halli Benasutti, Guankui Wang, Ernest V. Groman, Robert Scheinman, Laura Saba, Seyed Moein Moghimi (D) and Dmitri Simberg

Correction to: Nature Nanotechnology https://doi.org/10.1038/s41565-018-0344-3 published online 14 January 2019.

In the version of this Article originally published, a technical error led to Fig. 1a containing '!!!!!!!' above the scale bar. This has now been corrected in all versions of the Article. 